\title{
Positive KL-6 mucin expression combined with decreased membranous B-catenin expression indicates worse prognosis in colorectal carcinoma
}

\author{
WEI ZHANG ${ }^{1,2}$, WEI TANG ${ }^{2,3}$, YOSHINORI INAGAKI ${ }^{2}$, MING QIU $^{1}$, HUAN-LI XU $^{2,3}, \mathrm{XUN} \mathrm{LI}^{3}$, \\ YASUHIKO SUGAWARA ${ }^{2}$, HIROKAZU NAGAWA $^{4}$, MUNEHIRO NAKATA ${ }^{5}$ and NORIHIRO KOKUDO ${ }^{2}$
}

${ }^{1}$ Minimally Invasive Surgery Center, Changzheng Hospital, Second Military Medical University, Shanghai 200003, P.R. China; ${ }^{2}$ Hepato-Biliary-Pancreatic Surgery Division, Department of Surgery, Graduate School of Medicine, The University of Tokyo, Tokyo 113-8655, Japan; ${ }^{3}$ School of Pharmaceutical Sciences, Shandong University, Jinan 250012, P.R. China; ${ }^{4}$ Surgical Oncology Division, Department of Surgery, Graduate School of Medicine, The University of Tokyo, Tokyo 113-8655; ${ }^{5}$ Department of Applied Biochemistry, Tokai University, Kanagawa 259-1292, Japan

Received May 23, 2008; Accepted July 19, 2008

DOI: 10.3892/or_00000103

\begin{abstract}
Interaction of MUC1 with ß-catenin plays a significant role in tumor progression and invasion. However, the clinical significance of coexpression of MUC1 and subcellular B-catenin expression in colorectal carcinoma remains unclear. The present study evaluated the clinicopathological significance of their combined expression for predicting prognosis. Seventy-seven colorectal carcinomas were subjected to immunohistochemical staining with antiMUC1 KL-6 mucin and anti-ß-catenin monoclonal antibody. Positive KL-6 mucin expression was correlated with decreased membranous $B$-catenin expression $(\mathrm{P}=0.022)$, while no correlation was found between positive KL-6 expression and nuclear $\beta$-catenin expression $(\mathrm{P}=0.142)$. Preservation of membranous $\beta$-catenin expression was detected in 35 cases (45.5\%) and decreased membranous B-catenin expression was found in 42 cases $(54.5 \%)$. Negative KL-6 expression was detected in 31 cases (41.3\%) and positive expression was seen in 46 cases $(59.7 \%)$. Combined positive KL-6 expression and decreased membranous $\beta$-catenin expression was found in 30 patients $(39.0 \%)$, whose survival was significantly worse than that of patients with other expression patterns for these two molecules (53.3 vs. $84.4 \%, \mathrm{P}=0.005)$. Multivariate analysis showed that this combination was as an independent predictor of survival. We concluded that the combined pattern of positive KL-6 expression and decreased membranous B-catenin
\end{abstract}

Correspondence to: Dr Wei Tang, Hepato-Biliary-Pancreatic Surgery Division, Department of Surgery, Graduate School of Medicine, The University of Tokyo, 7-3-1 Hongo, Bunkyo-ku, Tokyo 113-8655, Japan

E-mail: tang-sur@h.u-tokyo.ac.jp

Key words: MUC1, KL-6, ß-catenin, colorectal carcinoma expression by colorectal carcinoma is a useful biomarker for distinguishing a subgroup of patients with a worse prognosis.

\section{Introduction}

$ß$-Catenin is a central component of the Wnt/ß-catenin signaling pathway, which has an important role in the development of colorectal carcinoma (1). B-Catenin was initially identified as a component of the adherens junction that is associated with E-cadherin, a cell-surface adhesion molecule and the connection between these two proteins is essential for strong cell-to-cell adhesion (2). Cytosolic Bcatenin is strictly regulated by ubiquitination-dependant proteolysis in a destruction complex comprising glycogen synthase kinase $3 \beta$ (GSK3ß), axin and the adenomatous polyposis coli gene (APC) (1). The APC gene is frequently inactivated in patients with familial adenomatous polyposis (FAP) and sporadic colorectal carcinoma by loss of heterozygosity $(\mathrm{LOH})$ or truncation mutations (3). Inactivation of APC is regarded as a crucial instigator of the pathogenesis of colorectal carcinoma (4), which results in the abrogation of $\beta$-catenin phosphorylation and stabilization in the cytoplasm. An increase of cytoplasmic $\beta$-catenin results in translocation into the nucleus, where it forms a complex with T-cell factor (TCF) or lymphocyte-enhancing factor-1 (LEF) and serves as a transcriptional co-activator that enhances the activity of downstream oncogenes, including c-myc (5) and cyclin D1 (6).

The pivotal functions of $\beta$-catenin are related to the cell membrane and the nucleus. Previous reports have indicated that the subcellular distribution of $\beta$-catenin is regulated by MUC1 overexpression (7-11). MUC1 is a well characterized transmembrane mucin that is expressed by secretory or polarized epithelium lining the luminal surface of the gastrointestinal, respiratory and reproductive tracts (12). MUC1 contains a large extracellular domain, a transmembrane domain and a cytoplasmic tail (CT), which harbors a serine rich motif that shows homology to sequences of E-cadherin and APC protein and functions as binding site for $\beta$-catenin (13). Aberrant 
overexpression of MUC1 is a common phenomenon in malignancies and plays a significant role in determining the outcome $(14,15)$. Overexpression of MUC1 has two effects on the subcellular distribution of $\beta$-catenin. First, MUC1 competitively blocks the binding of $\beta$-catenin to E-cadherin, so that $\beta$-catenin is recruited from the membrane adherent complex to the cytosol $(9,11)$. Second, MUC1 blocks the phosphorylation and degradation of $\beta$-catenin mediated by GSK3ß, which is involved in the cytosolic stabilization and nuclear translocation of $\beta$-catenin $(7,8)$.

Although in vitro experiments have suggested that interaction between MUC1 and B-catenin affects the intracellular distribution of $\beta$-catenin and may be an important factor for cancer promotion and progression, the clinical significance of the coexpression of MUC1 and Bcatenin is not yet clear. In the present study, an immunohistochemical analysis of the expression of MUC1 (using KL-6 monoclonal antibody) and the subcellular expression of ß-catenin was performed in colorectal carcinoma tissues. As a result, the clinical significance of combined expression of these molecules for predicting the prognosis of colorectal carcinoma was revealed.

\section{Materials and methods}

Study population. A total of 77 consecutive patients with colorectal carcinoma, who underwent radical resection at the Department of Surgery, Graduate School of Medicine, The University of Tokyo, between January 1991 and December 1992 were studied. This group comprised 51 men and 26 women aged $64.9 \pm 11.9$ years (mean \pm SD). All tumor specimens were classified according to the Japanese Classification of Colorectal Carcinoma produced by the Japanese Society for Cancer of the Colon and Rectum (16). The TNM system developed by the International Union Against Cancer was used for tumor staging (17). After clinicopathological analysis of the depth of invasion, patients were divided into two groups, which were a group with $\mathrm{m}, \mathrm{sm}$, or mp tumors and another group with ss, se, or si tumors (18).

Immunohistochemical staining. Paraffin-embedded tumor blocks were cut into $4-\mu \mathrm{m}$ thick sections and used to detect KL-6 and B-catenin expression separately. Slides were deparaffinized in xylene and rehydrated through a graded ethanol series. Endogenous peroxidase activity was blocked by incubation with $0.3 \%$ hydrogen peroxide/methanol for $30 \mathrm{~min}$. For antigen retrieval, sections were subjected to microwave heating twice for $10 \mathrm{~min}$ each in $10 \mathrm{mM}$ sodium citrate buffer (pH 6.0) (19). After blocking with goat serum for $30 \mathrm{~min}$, the sections were incubated with KL-6 monoclonal antibody (1:200 dilution; Eisai, Tokyo, Japan) for $60 \mathrm{~min}$ at room temperature, or with anti- $\beta$-catenin monoclonal antibody (1:200 dilution; BD Transduction Laboratories, Lexington, $\mathrm{KY}, \mathrm{USA}$ ) overnight at $4^{\circ} \mathrm{C}$. Then the sections were incubated with a biotinylated secondary antibody for $60 \mathrm{~min}$ and reaction products were detected by the steptavidin-biotinperoxidase complex method according to the manufacturer's instruction (Histofine SAB-PO kit, Nichirei, Tokyo, Japan). $\mathrm{DAB}$ was used as a chromogen and hematoxylin was used for counterstaining.
Evaluation of $K L-6$ and $\beta$-catenin staining. Expression of KL-6 and $\beta$-catenin in 10 random microscopic fields (or in the whole section if the slide contained $<10$ fields) was observed and recorded. KL-6 expression was evaluated, as described previously (20). Briefly, positive staining was recorded as brown granules in subcellular structures such as the luminal surface, cell membrane and cytoplasm. $\beta$-Catenin expression in adjacent normal colorectal epithelium served as the internal control. To grade membranous $\beta$-catenin expression, tumor tissues in which $>70 \%$ of the cells displayed membrane expression were defined as having preserved membranous $\beta$-catenin expression (18). In the case of nuclear $\beta$-catenin expression, nuclear staining of $>50 \%$ of the cells was defined as high nuclear B-catenin expression and nuclear staining of $<50 \%$ of the cells was considered to be low nuclear expression (21).

Statistical analysis. Statview 5.0J statistical software (Abacus Concepts, Berkeley, CA, USA) was used for statistical analysis. The $\chi^{2}$-test was employed to evaluate the relationship between KL-6/ß-catenin expression and clinicpathological features. Univariate survival analysis was performed according to the Kaplan-Meier product limit method and multivariate analysis was done according to the Cox proportional hazards model. In all analyses, a P-value of $<0.05$ was considered statistically significant.

\section{Results}

Subcellular $\beta$-catenin expression and relationship with clinicopathological features. All of the cells in normal colorectal epithelium showed strong membranous expression of $\beta$-catenin in the intercellular space, while nuclear staining was negative (Fig. 1A). In cancer tissues, there were various levels of B-catenin staining in the cytoplasm and statistical analysis showed that high cytoplasmic expression of B-catenin was related to the depth of tumor invasion (data not shown). As described above, cytoplasmic B-catenin is not functional, while the important functions of $\beta$-catenin are related to the cell membrane and nucleus. Therefore, we focused our analysis on these two subcellular regions. Membranous $\beta$-catenin expression was preserved in 35 cancers $(45.5 \%)$ (Fig. 1C), while it was decreased in 42 cancers $(54.5 \%)$ (Fig. 1E). Decreased membranous $\beta$-catenin expression was found to be significantly related to venous invasion $(\mathrm{P}=0.031$, Table I). Low nuclear $\beta$-catenin expression was detected in 32 cancers $(41.6 \%)$, while high nuclear expression was seen in 45 cancers $(58.4 \%)$. However, statistical evaluation of nuclear B-catenin expression did not reveal any significant differences in relation to the various clinicopathological features (Table I).

KL-6 expression and relationship with clinicopathological features. Normal colorectal epithelium cells showed no staining for KL-6 (Fig. 1B). Among 77 colorectal cancers, 43 tumors $(55.8 \%)$ were stained with KL-6. There were two distinct subcellular patterns of KL-6 expression by cancer tissue. The first was brown staining of the luminal surface of the glands (Fig. 1D). Since our previous study showed that this KL-6 pattern contributes little to the invasive potential of colon cancer and is easily removed by xylitol treatment $(20,22)$, the 
Table I. Relationship between KL-6/ß-catenin expression and clinicopathological parameters in colorectal carcinoma patients.

\begin{tabular}{|c|c|c|c|c|c|c|c|c|c|}
\hline \multirow{2}{*}{$\begin{array}{l}\text { Clinicopathological } \\
\text { Parameters (n) }\end{array}$} & \multicolumn{2}{|c|}{ KL-6 expression } & \multirow[b]{2}{*}{ P-value } & \multicolumn{2}{|c|}{ Membranous $\beta$-catenin } & \multirow[b]{2}{*}{ P-value } & \multicolumn{2}{|c|}{ Nuclear B-catenin } & \multirow[b]{2}{*}{ P-value } \\
\hline & Negative & Positive & & Preserved & Decreased & & Low & High & \\
\hline Age (years) & & & 0.643 & & & 0.859 & & & 0.197 \\
\hline$\leq 60(25)$ & $11(44.0)$ & $14(56.0)$ & & $11(44.0)$ & $14(56.0)$ & & $13(52.0)$ & $12(48.0)$ & \\
\hline$>60(52)$ & $20(38.5)$ & $32(61.5)$ & & $24(46.2)$ & $28(53.8)$ & & $19(36.5)$ & $33(63.5)$ & \\
\hline Gender & & & 0.471 & & & 0.567 & & & 0.559 \\
\hline Male (51) & $22(43.1)$ & $29(56.8)$ & & $22(43.1)$ & $29(56.9)$ & & $20(39.2)$ & $31(60.8)$ & \\
\hline Female (26) & $9(34.6)$ & $17(65.4)$ & & $13(50.0)$ & $13(50.0)$ & & $12(46.2)$ & $14(53.8)$ & \\
\hline Lymphatic invasion & & & $<0.001$ & & & 0.053 & & & 0.138 \\
\hline Negative (53) & $29(54.7)$ & $24(45.3)$ & & $28(52.8)$ & $25(47.2)$ & & $25(47.2)$ & $28(52.8)$ & \\
\hline Postive (24) & $2(8.3)$ & $22(91.7)$ & & $7(29.2)$ & $17(70.8)$ & & $7(29.2)$ & $17(70.8)$ & \\
\hline Venous invasion & & & 0.002 & & & 0.031 & & & 0.052 \\
\hline Negative (38) & $22(57.9)$ & $16(42.1)$ & & $22(57.9)$ & $16(42.1)$ & & $20(52.6)$ & $18(47.4)$ & \\
\hline Positive (39) & $9(23.1)$ & $30(76.9)$ & & $13(33.3)$ & $26(66.7)$ & & $12(30.8)$ & $27(69.2)$ & \\
\hline Lymph node metastasis & & & $<0.001$ & & & 0.278 & & & 0.363 \\
\hline Negative (41) & $31(73.8)$ & $11(26.2)$ & & $21(51.2)$ & $20(49.8)$ & & $19(46.3)$ & $22(53.7)$ & \\
\hline Positive (36) & $0(0)$ & $36(100)$ & & $14(38.9)$ & $22(61.1)$ & & $13(36.1)$ & $23(63.9)$ & \\
\hline Depth of invasion ${ }^{a}$ & & & 0.004 & & & 0.916 & & & 0.655 \\
\hline m, sm, mp (15) & $11(73.3)$ & $4(26.8)$ & & $7(46.7)$ & $8(53.3)$ & & $7(46.7)$ & $8(53.3)$ & \\
\hline ss, se, si (62) & $20(32.3)$ & $42(67.7)$ & & $28(45.2)$ & $34(54.8)$ & & $25(40.3)$ & $37(59.7)$ & \\
\hline TNM stage & & & $<0.001$ & & & 0.298 & & & 0.197 \\
\hline I, II (39) & $30(76.9)$ & $9(23.1)$ & & $20(51.3)$ & $19(48.7)$ & & $19(48.7)$ & $20(51.3)$ & \\
\hline III, IV (38) & $1(2.6)$ & $37(97.4)$ & & $15(39.5)$ & $23(60.5)$ & & $13(34.2)$ & $25(65.8)$ & \\
\hline
\end{tabular}

${ }^{a} \mathrm{~m}$, invasion of mucosa; sm, invasion of submucosa; mp, invasion of muscularis propria; ss, invasion of subserosa or subadventitia; se, invasion of serosa or adventitia and si, invasion of adjacent structures.

Table II. Correlation between KL-6 and B-catenin expression in colorectal carcinoma patients.

\begin{tabular}{|c|c|c|c|c|}
\hline & \multirow[b]{2}{*}{ Total } & \multicolumn{2}{|c|}{ KL-6 expression } & \multirow[b]{2}{*}{ P-value } \\
\hline & & Negative & Positive & \\
\hline \multicolumn{5}{|c|}{ Membranous $ß$-catenin expression } \\
\hline Preserved & 35 & $19(54.3)$ & $16(45.7)$ & 0.022 \\
\hline Decreased & 42 & $12(28.6)$ & $30(71.4)$ & \\
\hline \multicolumn{5}{|c|}{ Nuclear $ß$-catenin expression } \\
\hline Low & 32 & $16(50)$ & $16(50)$ & 0.142 \\
\hline High & 45 & $15(33.3)$ & $30(66.7)$ & \\
\hline
\end{tabular}

KL-6 mucin detected in this manner may be weakly attached to the luminal cell surface. It was thus considered to represent extracellular staining and was included with the negative group. The second pattern was brown cytoplasmic staining and/or circumferential cell membrane staining (Fig. 1F), which was defined as positive KL-6 expression. Negative expression was detected in 31 cancers $(41.3 \%)$, while positive expression was found in 46 cancers $(59.7 \%)$. As summarized in Table I, positive KL-6 expression was significantly related to the depth of tumor invasion, the presence of lymphatic or venous invasion, the presence of lymph node metastasis and a higher TNM stage.
Correlation of KL-6 expression and subcellular $\beta$-catenin expression. We analyzed the relationship between KL-6 expression and membranous or nuclear expression of $\beta$-catenin. As shown in Table II, there was a significant correlation between positive KL-6 expression and decreased membranous $\beta$-catenin expression ( $\mathrm{P}=0.022)$. However, KL-6 expression was not correlated with nuclear $B$-catenin expression $(\mathrm{P}=0.142)$.

Survival. Two patients were excluded from the analysis of survival because their deaths were not related to cancer. Using the Kaplan-Meier method, survival analysis was performed 


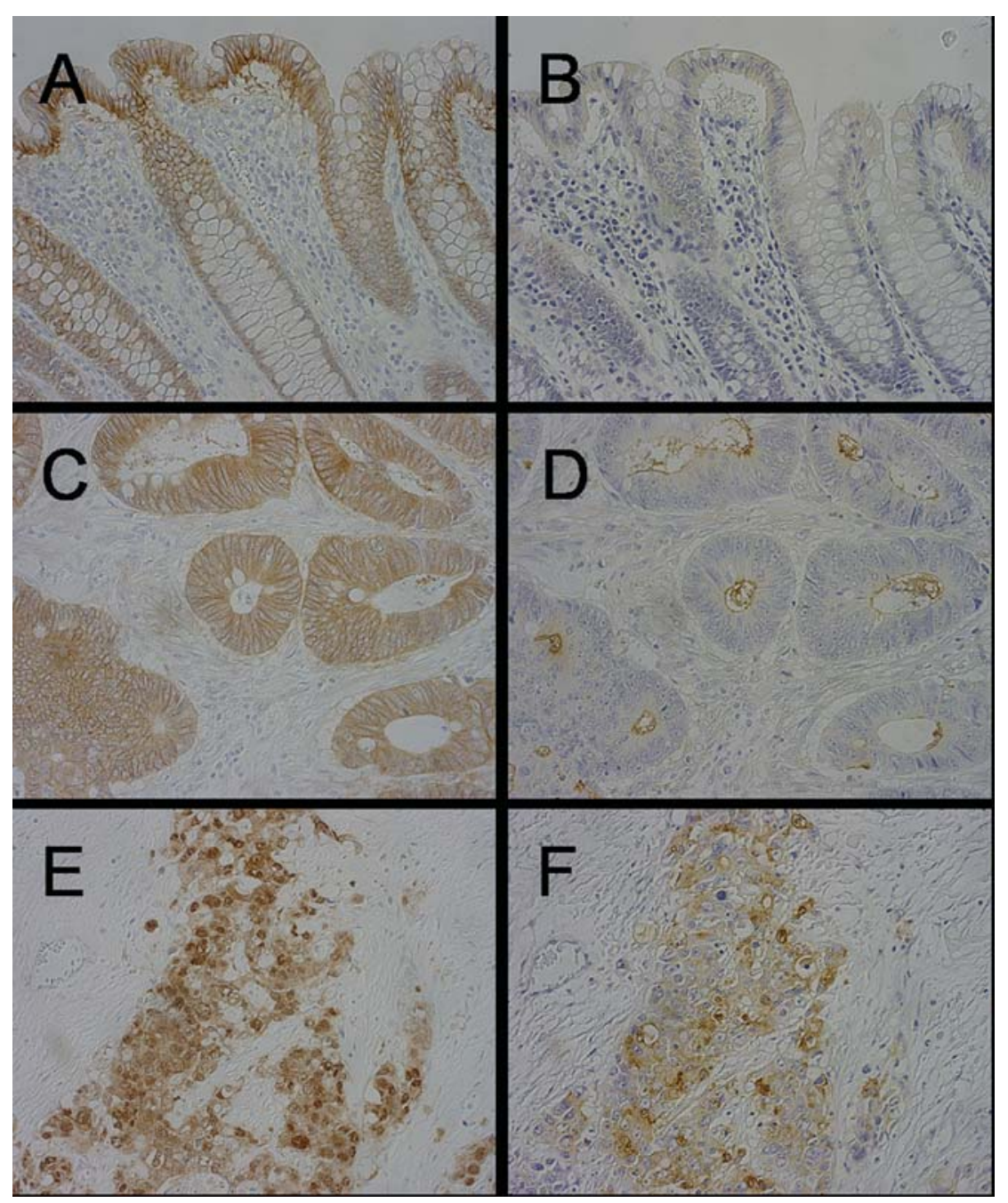

Figure 1. Immunohistochemical staining for B-catenin (A, C, E) and KL-6 (B, D, F) in colorectal tissues (magnification x200). (A) B-catenin is strongly expressed in the intercellular spaces of normal epithelium. (B) KL-6 is negative in normal colorectal epithelium. (C) Preserved membranous expression of $\beta$-catenin and (D) negative KL-6 expression (luminal surface) in colorectal carcinoma. (E) Decreased membranous expression of B-catenin (nuclear staining is also seen) and (F) positive KL-6 expression (cytoplasmic and circumferential) in colorectal carcinoma.

to investigate the potential prognostic impact of $\beta$-catenin and KL-6 expression by colorectal carcinoma. Both decreased membrane expression of $\beta$-catenin and positive KL- 6 expression were related to a worse prognosis and the associations were statistically significant $(\mathrm{P}=0.044$, Fig. 2A, and $\mathrm{P}=0.032$, Fig. $2 \mathrm{~B}$, respectively). In contrast, there was no significant difference of survival between low and high nuclear ß-catenin expression (data not shown). Because KL-6 positivity was correlated with decreased membranous B-catenin expression, we further analyzed the impact of combined expression on survival. This assessment revealed that the survival of patients with both negative KL-6 expression and preserved membranous $ß$-catenin expression was better than that of patients with either positive KL- 6 expression or decreased membranous $\beta$-catenin expression. On the other hand, patients with both positive KL-6 expression and decreased membranous $\beta$-catenin expression had the worst survival (Fig. 2C). There was a trend for patients with combined positive KL-6 expression and reduced membranous B-catenin expression to display the worst prognosis. We compared survival between patients with this pattern of expression and patients with any other pattern and found a significant difference between these two groups $(\mathrm{P}=0.005$, Fig. 2D).

Univariate and multivariate analyses. Univariate analysis showed that lymphatic invasion, lymph node metastasis, TNM stage, KL-6 expression, membranous $\beta$-catenin expression and the combination of positive KL-6 expression and reduced membranous $\beta$-catenin expressions were factors with a significant impact on overall survival (Table III). When multivariate analysis was done according to the Cox hazard model, the TNM stage was excluded because it incorporates the clinicopathological factor of lymph node metastasis (23). Neither positive KL-6 expression nor decreased membranous $ß$-catenin expression was a significant prognostic factor when assessed separately (data not shown). However, when their combined expression was analyzed, it was significantly related to a worse prognosis $(\mathrm{P}=0.008)$ and the risk ratio was 3.45 .

\section{Discussion}

The major roles of B-catenin are related to cell adhesion and the Wnt signal transduction pathway. The complex formed by 

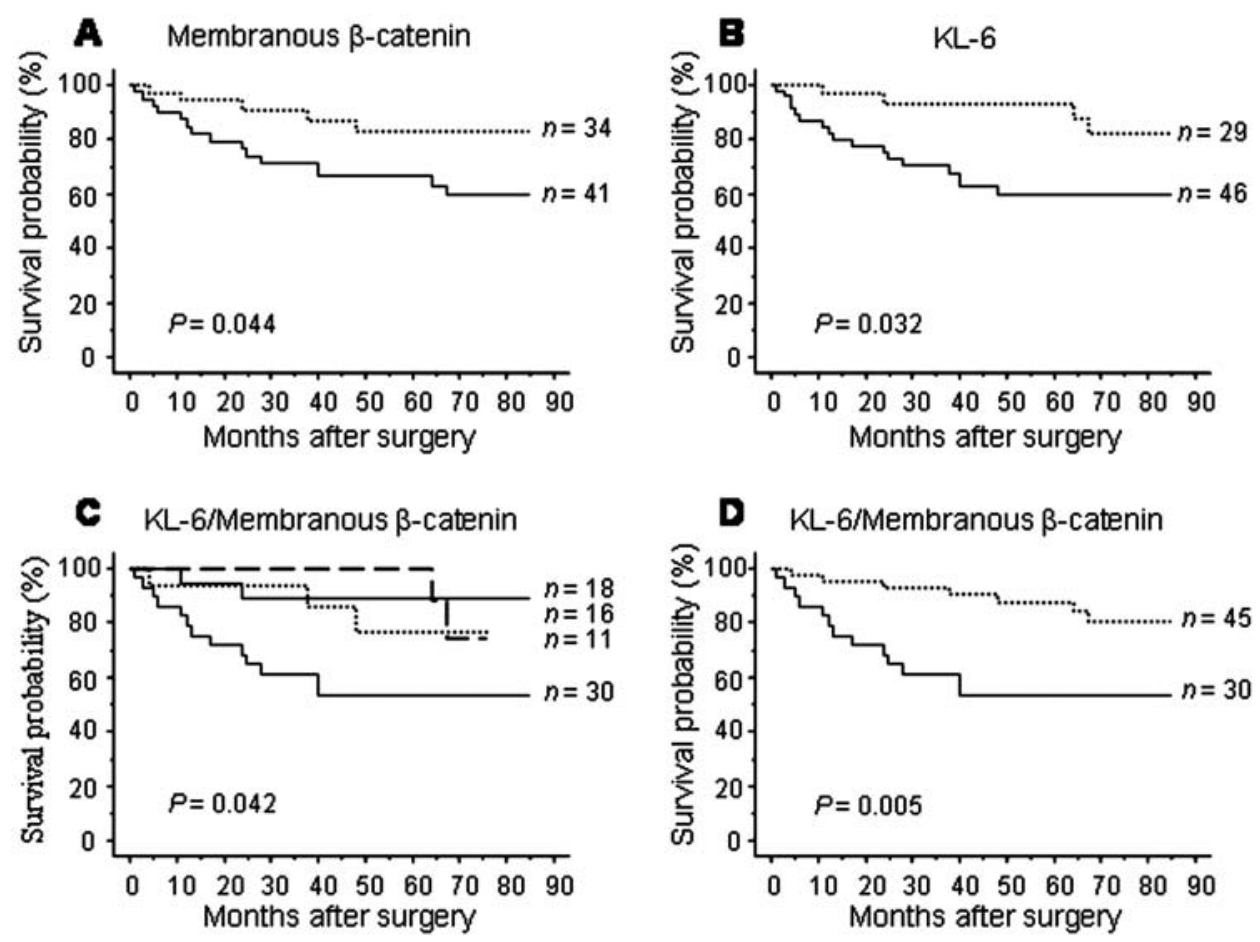

Figure 2. Kaplan-Meier analysis of survival in relation to $\beta$-catenin and KL-6 expression. (A) ß-catenin, preserved membranous expression (dotted line, $\mathrm{n}=34$ ) vs. decreased membranous expression (solid line, $n=41$ ). (B) KL-6, negative expression (dotted line, $n=29$ ) vs. positive expression (solid line, $n=46$ ). (C) Coexpression of KL-6 and membranous $\beta$-catenin: Negative KL-6/preserved membranous $\beta$-catenin (thin solid line, $\mathrm{n}=18$ ), positive KL-6/preserved membranous $\beta$-catenin (dotted line, $n=16$ ), negative KL-6/decreased membranous $\beta$-catenin (dashed line, $n=11$ ) and positive KL-6/decreased membranous $\beta$-catenin (thick solid line, $\mathrm{n}=30$ ); (D) combined KL-6 expression and decreased membranous $\beta$-catenin expression (solid line, $\mathrm{n}=30$ ) vs. all other patterns (dotted line, $\mathrm{n}=45$ ).

Table III. Univariate analysis of the survival of colorectal carcinoma patients.

\begin{tabular}{|c|c|c|}
\hline Category & Survival rate $(\%)$ & P-value \\
\hline $\begin{array}{l}\text { Age } \\
\leq 60 \text { vs. }>60\end{array}$ & 68 vs. 74 & 0.708 \\
\hline $\begin{array}{l}\text { Gender } \\
\text { Male vs. female }\end{array}$ & 73.5 vs. 69.2 & 0.823 \\
\hline $\begin{array}{l}\text { Lymphatic invasion } \\
\text { Positive vs. negative }\end{array}$ & 56.5 vs. 78.9 & 0.007 \\
\hline $\begin{array}{l}\text { Venous invasion } \\
\text { Positive vs. negative }\end{array}$ & 65.8 vs. 78.4 & 0.116 \\
\hline $\begin{array}{l}\text { Lymph node metastasis } \\
\text { Positive vs. negative }\end{array}$ & 61.1 vs. 82.1 & 0.016 \\
\hline $\begin{array}{l}\text { Depth of invasion } \\
\text { ss, se, si vs. m, sm, mp }\end{array}$ & 69.4 vs. 84.6 & 0.331 \\
\hline $\begin{array}{l}\text { TNM stage } \\
\text { III, IV vs. I, II }\end{array}$ & 63.2 vs. 81.1 & 0.037 \\
\hline $\begin{array}{l}\text { KL-6 } \\
\text { Positive vs. negative }\end{array}$ & 63.0 vs. 86.2 & 0.032 \\
\hline $\begin{array}{l}\text { B-catenin } \\
\text { Decreased membranous vs. preserved membranous }\end{array}$ & 61.0 vs. 85.3 & 0.044 \\
\hline $\begin{array}{l}\text { B-catenin } \\
\text { Nuclear high vs. nuclear low }\end{array}$ & 72.1 vs. 71.9 & 0.760 \\
\hline $\begin{array}{l}\text { Positive KL-6/decreased membranous } \beta \text {-catenin } \\
\text { vs. others }\end{array}$ & 53.3 vs. 84.4 & 0.005 \\
\hline
\end{tabular}


B-catenin and E-cadherin is important for cell-cell adhesion and breakdown of the E-cadherin/ß-catenin complex results in loss of the 'invasion suppressor system.' Thus, decreased expression of $\beta$-catenin has been observed in many tumors (24-26). In patients with colorectal carcinoma, decreased or absent membrane expression of $\beta$-catenin has been reported to play a key role in tumor progression and metastasis $(27,28)$. The present study also revealed a significant relation of such expression to a worse prognosis. The survival rate was $85.3 \%$ for patients with preserved membranous $\beta$-catenin expression vs. only $61.0 \%$ for those with decreased $B$-catenin expression (Fig. 2A). This result is consistent with the conclusion that primary colorectal carcinoma with a decreased level of membrane $\beta$-catenin expression is associated with a lower overall survival rate $(27,28)$. Nuclear $\beta$-catenin expression is frequently detected in colorectal carcinoma (29-31), but its significance as a prognostic indicator is still controversial. Wong et al (29) reported that a higher nuclear $\beta$-catenin staining score was related to the presence of lymph node metastasis and a poor prognosis, while Horkko et al (30) and Gunther et al (31) did not find any relationship between nuclear ß-catenin staining and the prognosis. The present study also found no relationship between nuclear B-catenin expression and either clinopathological features (Table I) or the prognosis (Table III). This suggests that nuclear B-catenin-TCF/LEF signaling may be crucial for the early stage of malignant transformation, while its contribution to the development and invasion of colorectal carcinoma is limited (31).

The epitope recognized by KL-6 monoclonal antibody is MUC1, a mucin-bearing sialylated carbohydrate (32). In normal colorectal tissue, there was no expression of KL-6 mucin (Fig. 1B), while colorectal carcinoma tissue showed positive expression in the cytoplasm and/or circumferential membrane expression (Fig. 1F), which represented the aberrant pattern of MUC1 expression. It has been proposed that aberrant MUC1 expression has an anti-adhesive effect by interfering with cell-to-cell interaction, thereby facilitating detachment of tumor cells from the primary lesion $(22,33)$. In the present study, KL-6 expression was found to be significantly related to several clinicopathological features that reflect tumor progression and invasion, such as the depth of invasion, presence of lymphatic and venous invasion, presence of lymph node metastasis and higher TNM stage (Table I), indicating that tumors with KL-6 expression were more likely to show deeper invasion, metastasize to lymph nodes and progress to a more advanced stage.

Interference with cell-to-cell adhesion is mediated by the long extracellular domain of MUC1 through a process of steric hindrance (33). In recent years, several in vitro experiments have confirmed that overexpression of MUC1 also modulates the intracellular distribution and signal transduction of $B$ catenin (7-11), suggesting that an interaction between MUC1 and $B$-catenin might influence tumor progression. However, the present clinical study found no correlation between KL-6 expression and nuclear $\beta$-catenin expression (Table II). In contrast, there was a significant correlation between aberrant KL-6 expression and decreased membranous B-catenin expression (Table II), indicating that KL-6 might mainly modulate the function of $\beta$-catenin at the adherens junction. Schroeder et al (11) detected the colocalization of MUC1 and ß-catenin in invading cell membranes, suggesting a novel pathway of $\beta$-catenin-induced tumorigenesis that involved recruitment of ß-catenin away from the adherens complex. Li et al (9) also confirmed that overexpression of MUC1 competitively decreased the binding of $\beta$-catenin to E-cadherin, thus interfering with cell adhesion and having an antiadhensive effect on tumor cells. When the correlation between clinicopathological features and the combination of positive KL- 6 expression and decreased membranous Bcatenin was analyzed, this combination was related to deeper tumor invasion, the presence of lymphatic and venous invasion, positive lymph node metastasis and a higher TNM stage (data not shown). Survival analysis showed that patients with both positive KL-6 expression and decreased membranous ß-catenin expression had worse survival than patients with either positive KL-6 expression or decreased membranous $\beta$-catenin expression alone (Fig. 2C). The overall survival rate of the former group was $53.3 \%$, which was lower than that of the latter group $(84.4 \%$, Table III). These findings suggest that modulation of membranous B-catenin expression by KL-6 facilitates the progression of cancer and that the synergetic effect of combined expression is related to aggressive invasion. Univariate and multivariate analysis both showed that combined KL-6 expression and decreased membranous $\beta$-catenin expression (rather than the detection of either pattern alone) was an independent predictor of the prognosis. Thus, only this pattern of expression seems to be clinically useful.

In conclusion, immunohistochemical analysis of KL-6 and $ß$-catenin expression by colorectal cancer showed that positive expression of KL- 6 was related to decreased membranous expression of $\beta$-catenin. Combined detection of KL-6 positivity and decreased membranous $\beta$-catenin expression was related to a significantly worse prognosis. This simple method of evaluating the expression of KL- 6 and $\beta$-catenin seems to have clinical significance for predicting the prognosis and deciding the optimal treatment plan.

\section{Acknowledgements}

This study was supported by Grants-in-aid from the Ministry of Education, Science, Sports and Culture of Japan and partly supported by the Japan-China Sasakawa Medical Fellowship.

\section{References}

1. Behrens J: The role of the Wnt signaling pathway in colorectal tumorigenesis. Biochem Soc Trans 33: 672-675, 2005.

2. Ozawa M, Baribault $\mathrm{H}$ and Kemler R: The cytoplasmic domain of the cell adhesion molecule uvomorulin associates with three independent proteins structurally related in different species. EMBO J 8: 1711-1717, 1989.

3. Fuchs SY, Ougolkov AV, Spiegelman VS and Minamoto T: Oncogenic beta-catenin signaling networks in colorectal cancer. Cell Cycle 4: 1522-1539, 2005.

4. Potter JD: Colorectal cancer: molecules and populations. J Natl Cancer Inst 91: 916-932, 1999.

5. He TC, Sparks AB, Rago C, Hermeking H, Zawel L, da Costa LT, Morin PJ, Vogelstein B and Kinzler KW: Identification of c-MYC as a target of the APC pathway. Science 281: 1509-1512, 1998.

6. Tetsu O and McCormick F: Beta-catenin regulates expression of cyclin D1 in colon carcinoma cells. Nature 398: 422-426, 1999.

7. Huang L, Chen D, Liu D, Yin L, Kharbanda S and Kufe D: MUC1 oncoprotein blocks glycogen synthase kinase 3betamediated phosphorylation and degradation of beta-catenin. Cancer Res 65: 10413-10422, 2005. 
8. Wen Y, Caffrey TC, Wheelock MJ, Johnson KR and Hollingsworth MA: Nuclear association of the cytoplasmic tail of MUC1 and beta-catenin. J Biol Chem 278: 38029-38039, 2003.

9. Li Y, Bharti A, Chen D, Gong J and Kufe D: Interaction of glycogen synthase kinase 3beta with the DF3/MUC1 carcinomaassociated antigen and beta-catenin. Mol Cell Biol 18: 7216-7224, 1998.

10. Huang L, Ren J, Chen D, Li Y, Kharbanda S and Kufe D: MUC1 cytoplasmic domain coactivates Wnt target gene transcription and confers transformation. Cancer Biol Ther 2: 702-706, 2003.

11. Schroeder JA, Adriance MC, Thompson MC, Camenisch TD and Gendler SJ: MUC1 alters beta-catenin-dependent tumor formation and promotes cellular invasion. Oncogene 22: 1324-1332, 2003

12. Hollingsworth MA and Swanson BJ: Mucins in cancer: protection and control of the cell surface. Nat Rev Cancer 4: 45-60, 2004.

13. Byrd JC and Bresalier RS: Mucins and mucin binding proteins in colorectal cancer. Cancer Metastasis Rev 23: 77-99, 2004.

14. Rahn JJ, Dabbagh L, Pasdar M and Hugh JC: The importance of MUC1 cellular localization in patients with breast carcinoma: an immunohistologic study of 71 patients and review of the literature. Cancer 91: 1973-1982, 2001.

15. Cao Y, Schlag PM and Karsten U: Immunodetection of epithelial mucin (MUC1, MUC3) and mucin-associated glycotopes (TF, Tn, and sialosyl-Tn) in benign and malignant lesions of colonic epithelium: apolar localization corresponds to malignant transformation. Virchows Arch 431: 59-66, 1997.

16. Japanese Society for Cancer of the Colon and Rectum. In Japanese classification of colorectal carcinoma (ed). 1st English edition. Kanehara, Tokyo, 1997.

17. Sobin LH and Wittekind CH: International Union Against Cancer (UICC). In: TNM classification of the malignant tumor (eds). 5th edition. Wiley-Liss Publications, New York, 1997.

18. Maruyama K, Ochiai A, Akimoto S, Nakamura S, Baba S, Moriya Y and Hirohashi S: Cytoplasmic beta-catenin accumulation as a predictor of hematogenous metastasis in human colorectal cancer. Oncology 59: 302-309, 2000.

19. Tang W, Miki K, Kokudo N, Sugawara Y, Imamura H, Minagawa M, Yuan LW, Ohnishi S and Makuuchi M: Desgamma-carboxy prothrombin in cancer and non-cancer liver tissue of patients with hepatocellular carcinoma. Int J Oncol 22: 969-975, 2003

20. Guo Q, Tang W, Inagaki Y, Midorikawa Y, Kokudo N, Sugawara Y, Nakata M, Konishi T, Nagawa $\mathrm{H}$ and Makuuchi M: Clinical significance of subcellular localization of KL-6 mucin in primary colorectal adenocarcinoma and metastatic tissues. World J Gastroenterol 12: 54-59, 2006.

21. Baldus SE, Monig SP, Huxel S, Landsberg S, Hanisch FG Engelmann K, Schneider PM, Thiele J, Holscher AH and Dienes HP: MUC1 and nuclear beta-catenin are coexpressed at the invasion front of colorectal carcinomas and are both correlated with tumor prognosis. Clin Cancer Res 10: 2790-2796, 2004.
22. Guo Q, Tang W, Inagaki Y, Kokudo N, Sugawara Y, Karako H, Nakata M and Makuuchi M: Subcellular localization of KL-6 mucin in colorectal carcinoma cell lines: Association with metastatic potential and cell morphology. Oncol Rep 17: 1057-1060, 2007

23. Tang W, Kokudo N, Sugawara Y, Guo Q, Imamura H, Sano K, Karako H, Qu X, Nakata M and Makuuchi M: Des-gammacarboxyprothrombin expression in cancer and/or non-cancer liver tissues: association with survival of patients with resectable hepatocellular carcinoma. Oncol Rep 13: 25-30, 2005.

24. Choi YS, Shim YM, Kim SH, Son DS, Lee HS, Kim GY, Han J and Kim J: Prognostic significance of E-cadherin and beta-catenin in resected stage I non-small cell lung cancer. Eur J Cardiothorac Surg 24: 441-449, 2003

25. Ohno T, Aihara R, Kamiyama Y, Mochiki E, Asao T and Kuwano H: Prognostic significance of combined expression of MUC1 and adhesion molecules in advanced gastric cancer. Eur J Cancer 42: 256-263, 2006.

26. van Oort IM, Tomita K, van Bokhoven A, Bussemakers MJ, Kiemeney LA, Karthaus HF, Witjes JA and Schalken JA: The prognostic value of E-cadherin and the cadherin-associated molecules alpha-, beta-, gamma-catenin and p120(ctn) in prostate cancer specific survival: A long-term follow-up study. Prostate 67: 1432-1438, 2007.

27. Fernebro E, Bendahl PO, Dictor M, Persson A, Fernö M and Nilbert M: Immunohistochemical patterns in rectal cancer: application of tissue microarray with prognostic correlations. Int J Cancer 111: 921-928, 2004

28. Han SA, Chun H, Park CM, Kang SJ, Kim SH, Sohn D, Yun SH and Lee WY: Prognostic significance of beta-catenin in colorectal cancer with liver metastasis. Clin Oncol (R Coll Radiol) 18: 761-767, 2006.

29. Wong SC, Lo ES, Lee KC, Chan JK and Hsiao WL: Prognostic and diagnostic significance of beta-catenin nuclear immunostaining in colorectal cancer. Clin Cancer Res 10: 1401-1408, 2004.

30. Horkko TT, Klintrup K, Makinen JM, Napankangas JB, Tuominen HJ, Makela J, Karttunen TJ and Makinen MJ Budding invasive margin and prognosis in colorectal cancer - no direct association with beta-catenin expression. Eur J Cancer 42: 964-971, 2006

31. Gunther K, Brabletz T, Kraus C, Dworak O, Reymond MA, Jung A, Hohenberger W, Kirchner T, Kockerling F and Ballhausen WG: Predictive value of nuclear beta-catenin expression for the occurrence of distant metastases in rectal cancer. Dis Colon Rectum 41: 1256-1261, 1998.

32. Kohno N, Akiyama M, Kyoizumi S, Hakoda M, Kobuke K and Yamakido M: Detection of soluble tumor-associated antigens in sera and effusions using novel monoclonal antibodies, KL-3 and KL-6, against lung adenocarcinoma. Jpn J Clin Oncol 18: 203-216, 1988.

33. Wesseling $\mathbf{J}$, van der Valk SW and Hilkens J: A mechanism for inhibition of E-cadherin-mediated cell-cell adhesion by the membrane-associated mucin episialin/MUC1. Mol Biol Cell 7: 565-577, 1996. 